\title{
Distribution of Cardioembolic Stroke: A Cohort Study
}

\author{
Ramon Pierik $^{a}$ Ale Algra $^{b}$ Ewoud van Dijk ${ }^{c}$ Michiel E. Erasmus ${ }^{d}$ \\ Isabella C. van Gelder ${ }^{\mathrm{e}}$ Peter J. Koudstaal ${ }^{\mathrm{f}}$ Gert-Jan R. Luijckx ${ }^{\mathrm{g}}$ \\ Paul J. Nederkoorn ${ }^{h}$ Robert J. van Oostenbrugge ${ }^{i}$ Ynte M. Ruigrok $^{j}$ \\ Thomas W.L. Scheeren ${ }^{k}$ Maarten Uyttenboogaart ${ }^{g}$ Marieke C. Visser ${ }^{h}$ \\ Marieke J.H. Wermer' Walter M. van den Bergh ${ }^{a}$ on behalf of the Parelsnoer \\ Institute-Cerebrovascular Accident Study Group
}

\begin{abstract}
a Department of Critical Care, University Medical Center Groningen, University of Groningen, Groningen, The Netherlands; ${ }^{b}$ Department of Neurology and Neurosurgery, Brain Center Rudolf Magnus and the Julius Center for Health Sciences and Primary Care, University Medical Center Utrecht, Utrecht, The Netherlands; ' ${ }^{D}$ Department of Neurology, Radboud University Medical Center, Nijmegen, The Netherlands; 'Department of Cardiac Surgery, University Medical Center Groningen, University of Groningen, Groningen, The Netherlands; ${ }^{\text {e Department of }}$ Cardiology, University Medical Center Groningen, University of Groningen, Groningen, The Netherlands; ${ }^{f}$ Department of Neurology, Erasmus University Medical Center, Rotterdam, The Netherlands; ${ }^{9}$ Department of Neurology, University Medical Center Groningen, University of Groningen, Groningen, The Netherlands; ' Department of Neurology, Amsterdam University Medical Centers, University of Amsterdam, Amsterdam, The Netherlands; iDepartment of Neurology, Maastricht University Medical Center, Maastricht, The Netherlands; ${ }^{j}$ Department of Neurology and Neurosurgery, Brain Center Rudolf Magnus, University Medical Center Utrecht, Utrecht, The Netherlands; ${ }^{\mathrm{k}}$ Department of Anesthesiology, University Medical Center Groningen, University of Groningen, Groningen, The Netherlands; 'Department of Neurology, Leiden University Medical Center, Leiden, The Netherlands
\end{abstract}

\section{Keywords}

Stroke · Posterior circulation brain infarction · Intracranial embolism and thrombosis - Cardiovascular diseases

\begin{abstract}
Background: A cardiac origin in ischemic stroke is more frequent than previously assumed, but it is not clear which patients benefit from cardiac work-up if obvious cardiac pathology is absent. We hypothesized that thromboembolic stroke with a cardiac source occurs more frequently in the posterior circulation compared with thromboembolic stroke of another etiology. Methods: We performed a multicenter
\end{abstract}

\begin{tabular}{ll}
\hline KARGER & ( ) 2020 The Author(s) Karger \\
& Published by S. Karger AG, Basel Open access \\
karger@karger.com & This article is licensed under the Creative Commons Attribution- \\
NonCommercial-NoDerivatives 4.0 International License (CC BY- \\
Nww.karger.com/ced \\
Nsage and distribution for commercial purposes as well as any dis- \\
tribution of modified material requires written permission.
\end{tabular}

observational study in 3,311 consecutive patients with ischemic stroke who were enrolled in an ongoing prospective stroke registry of 8 University hospitals between September 2009 and November 2014 in The Netherlands. In this initiative, the so-called Parelsnoer Institute-Cerebrovascular Accident Study Group, clinical data, imaging, and biomaterials of patients with stroke are prospectively and uniformly collected. We compared the proportions of posterior stroke location in patients with a cardiac stroke source with those with another stroke etiology and calculated risk ratios (RR) with corresponding $95 \% \mathrm{Cl}$ with Poisson regression analyses. To assess which patient or disease characteristics were most strongly associated with a cardiac etiology in patients
Walter M. van den Bergh, MD, PhD

Department of Critical Care

University Medical Center Groningen, Room BA.49

PO Box 30001, NL-9700 RB Groningen (The Netherlands)

E-Mail w.m.van.den.bergh@umcg.nl 
with ischemic stroke, we performed a stepwise backward regression analysis. Results: For the primary aim, 1,428 patients were eligible for analyses. The proportion of patients with a posterior stroke location among patients with a cardiac origin of their stroke (28\%) did not differ statistically significant to those with another origin (25\%), age and sex adjusted RR 1.16; $95 \% \mathrm{Cl} 0.96-1.41$. For the secondary aim, 1,955 patients were eligible for analyses. No recent history of smoking, no hyperlipidemia, coronary artery disease, a higher age, and a higher National Institutes of Health Stroke Scale (NIHSS) score were associated with a cardiac etiology of ischemic stroke. Conclusions: We could not confirm our hypothesis that thromboembolic stroke localized in the posterior circulation is associated with a cardioembolic source of ischemic stroke, and therefore posterior stroke localization on itself does not necessitate additional cardiac examination. The lack of determinants of atherosclerosis, for example, no recent history of smoking and no hyperlipidemia, coronary artery disease, a higher age, and a higher NIHSS score are stronger risk factors for a cardiac source of ischemic stroke.

\section{(C) 2020 The Author(s)}

Published by S. Karger AG, Basel

\section{Introduction}

Based on studies demonstrating that prolonged and continuously heart rhythm monitoring in patients at risk of atrial fibrillation (AF) and in patients with cryptogenic stroke, it is now recognized that cardiac embolism is a more frequent cause for thromboembolic stroke than previously thought [1-3]. A pragmatic approach in which patients with an ischemic stroke of undetermined source were treated with rivaroxaban, an oral factor Xa inhibitor, was studied in a clinical trial showing that rivaroxaban was not superior to aspirin with regard to the prevention of recurrent stroke and was associated with a higher risk of bleeding [4]. A more recent study showed that the oral direct thrombin inhibitor dabigatran was also not superior to aspirin in preventing recurrent stroke with more clinically relevant nonmajor bleeding events in the dabigatran group [5]. Therefore, proper selection of patients with a high chance of cardioembolic stroke for which secondary prevention with an oral factor Xa inhibitor will be beneficial is needed.

Phenotyping of patients with an ischemic stroke of undetermined source with high risk of a cardiac etiology, for example, AF or patent foramen ovale (PFO), may increase the efficacy of additional cardiac workup. Although several characteristics, including strokes in different vascular territories, isolated aphasia [6], increased age, male sex, heart failure, and pulmonary disease, are associated with $\mathrm{AF}$ occurrence, no clear evidence exists which (combination of) risk factors identify the patients who would derive the most clinical benefit from detection of AF by prolonged monitoring.

We found in a single-hospital retrospective observational study in 7,454 consecutive patients after cardiac surgery in which we assumed that all perioperative thromboembolic strokes are of cardiac origin, and that thromboembolic stroke after cardiac surgery occurs twice as often in the posterior cerebral circulation compared with ischemic strokes in the general population [7]. This risk was further increased in presence of multiple strokes. If these findings are confirmed in general stroke cohorts, ischemic stroke located in the posterior cerebral circulation may lower the threshold for initiating additional cardiac diagnostic studies in order to point toward a cardiac source in these patients.

The primary aim of the current study was to confirm in a large independent cohort that strokes with a cardiac etiology are more often located in the posterior circulation area than strokes of another etiology. The secondary aim was to study the association of clinical characteristics with cardioembolic strokes in patients with ischemic stroke.

\section{Methods}

Study Population and Definition of Stroke

We included patients with cerebral ischemia, including both ischemic stroke and transient ischemic attack (TIA), who were enrolled in an ongoing prospective registry of 8 University hospitals between September 2009 and November 2014 in The Netherlands [8]. In this initiative, the so-called Parelsnoer Institute-Cerebrovascular Accident Study Group, clinical data, imaging, and biomaterials of patients with stroke are prospectively and uniformly collected. We approached all eligible patients, or a next of kin when patients were unconsciousness or mentally incompetent, for informed consent within the first 3 months after the event. The Ethics Committees of all participating centers approved the study, and all patients provided written informed consent.

Ischemic stroke and TIA were defined according to the World Health Organization criteria for stroke [9]. We further classified ischemic stroke into specific subtypes according to Trial of Org 10172 in Acute Stroke Treatment (TOAST) classification: large artery atherosclerosis, small vessel disease, cardioembolic stroke, other determined cause, and undetermined cause [10]. Patients determined with definitive cardioembolic stroke according to the A-S-C-O classification were also considered as cardioembolic stroke if TOAST classification was missing or undetermined [11]. Cardioembolic stroke was not further differentiated into AF or otherwise.

We categorized stroke localization based on the modified Oxfordshire method that classifies the infarcts based on their ana-
98

Cerebrovasc Dis 2020;49:97-104 DOI: $10.1159 / 000505616$
Pierik et al. 
Table 1. Baseline characteristics of 2,334 patients based on cardioembolism $(n=578)$ as source for ischemic stroke or TIA or $\operatorname{not}(n=1,756)$

\begin{tabular}{|c|c|c|c|}
\hline Variable & $\begin{array}{l}\text { Cardioembolism } \\
(n=578), n(\%)\end{array}$ & $\begin{array}{l}\text { No cardioembolism } \\
(n=1,756), n(\%)\end{array}$ & $p$ value \\
\hline $\mathrm{BMI}$, mean $\pm \mathrm{SD}^{*}$ & $26 \pm 5$ & $26 \pm 4$ & 0.98 \\
\hline NIHSS score, median (IQR)* & $3(1-8)$ & $2(0-5)$ & $<0.001$ \\
\hline Age, years, mean \pm SD & $70 \pm 15$ & $65 \pm 14$ & $<0.001$ \\
\hline Age category per decade & & & $<0.001$ \\
\hline $0-20$ & $4(1)$ & $8(1)$ & \\
\hline $21-30$ & $8(1)$ & $12(1)$ & \\
\hline $31-40$ & $17(3)$ & $61(3)$ & \\
\hline $41-50$ & $42(7)$ & $177(10)$ & \\
\hline $51-60$ & $62(11)$ & $342(19)$ & \\
\hline $61-70$ & $141(24)$ & $530(30)$ & \\
\hline $71-80$ & $173(30)$ & $416(24)$ & \\
\hline $81-90$ & $113(20)$ & $187(11)$ & \\
\hline $91-100$ & $18(3)$ & $23(1)$ & \\
\hline Gender, male & $352(61)$ & $1,043(59)$ & 0.52 \\
\hline Hypertension & $342(60)$ & $986(56)$ & 0.19 \\
\hline Hyperlipidemia & $188(33)$ & $655(37)$ & 0.08 \\
\hline Diabetes mellitus & $97(17)$ & $289(16)$ & 0.72 \\
\hline Coronary artery disease & $139(24)$ & $288(16)$ & $<0.001$ \\
\hline Peripheral arterial occlusion disease & $50(9)$ & $170(10)$ & 0.57 \\
\hline Recent ( $<6$ month) history of smoking & $94(16)$ & $572(33)$ & $<0.001$ \\
\hline Use of oral anticoagulants (prior to stroke) & $85(15)$ & $173(10)$ & $<0.001$ \\
\hline Use of antiplatelets (prior to stroke) & $201(35)$ & $728(41)$ & 0.012 \\
\hline History of migraine* & $51(9)$ & $197(11)$ & 0.095 \\
\hline $\mathrm{AF}$ (paroxysmal) & $292(51)$ & $54(3)$ & $<0.001$ \\
\hline
\end{tabular}

* $>3 \%$ missing's (BMI 24\%, NIHSS 18\%, and migraine 35\% unknown).

TIA, transient ischemic attack; BMI, body mass index; NIHSS, National Institutes of Health Stroke Scale; AF, atrial fibrillation.

tomic distribution into 4 groups: total anterior circulation infarcts, partial anterior circulation infarcts, posterior circulation infarcts, and lacunar infarcts [12]. Patients with infarcts in multiple territories that involved the posterior cerebral circulation were considered posterior circulation infarct patients in the primary analysis.

\section{Statistical Analyses}

We compared the proportion of patients with a posterior stroke location among patients with a cardiac origin of their stroke and those with another origin and calculated a risk ratio (RR) with corresponding 95\% CI. In additional Poisson regression analyses, we assessed the influence of adjustment of the crude RRs for potential confounders [13]. Bivariable analyses were performed with all patient and baseline characteristics mentioned in Table 1 with exception of AF as this already assumes a history of AF and a cardiac etiology in the workup of the Parelsnoer Institute-Cerebrovascular Accident Study Group. If the tested variable changed the crude RR of posterior stroke $>10 \%$ the variable was used in the subsequent multivariable analyses. A sensitivity analysis was performed in which patients with a TIA were excluded.

To assess if patient or disease characteristics were associated with a cardiac etiology in patients with ischemic stroke, we performed a stepwise logistic regression analysis using a $p$ value of
0.10 to eliminate variables with the backward method. The full model consisted of patient and baseline characteristics mentioned in Table 1, again with exception of AF. Use of oral anticoagulants and antiplatelets was excluded because of confounding by indication. Variables with $>20 \%$ missing's were also excluded. Thereafter, a risk score was calculated using the beta's of the remaining variables in the model. Discrimination of the risk score was calculated with the concordance (c) statistic and corresponding receiver operating characteristic curve.

Missing values were not imputed in our analyses.

Data Availability

The data that support the findings of this study are available from the corresponding author upon reasonable request.

\section{Results}

The database contained 3,311 patients with an ischemic stroke or TIA. For the primary aim, 1,212 patients were excluded because stroke localization was undeter- 
Fig. 1. Flowchart of included patients. TIA, transient ischemic attack

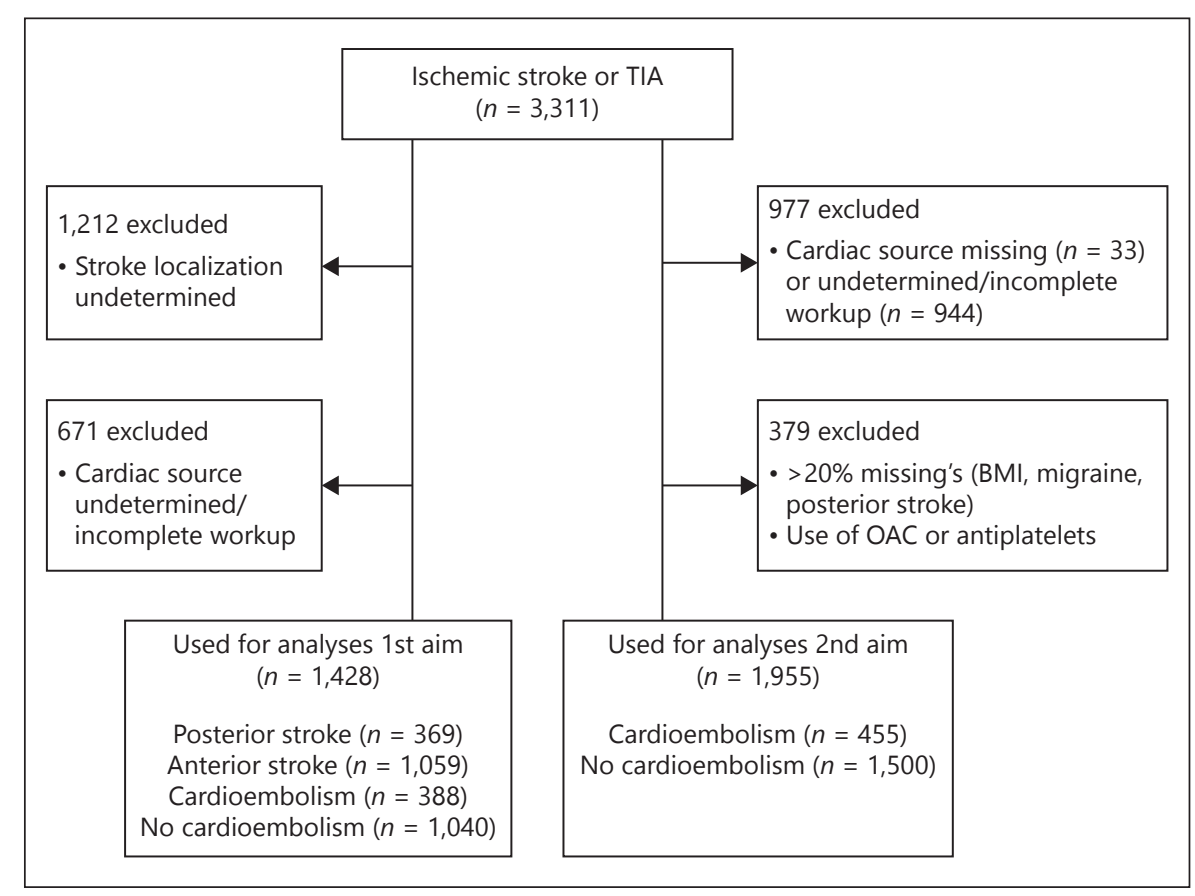

mined, and another 671 patients were excluded because stroke etiology was undetermined leaving 1,428 eligible patients for analyses. For the secondary aim, 977 patients were excluded because stroke etiology was undetermined, for example, incomplete cardiac work-up. Another 379 patients were excluded because variables contain too many missing's or patients already used anticoagulants leaving 1,955 eligible patients for analyses (Fig. 1).

In $578(18 \%)$ patients, the source of cerebral ischemia was cardioembolism (Table 2). Patients with cardioembolic stroke had increased age, a higher National Institutes of Health Stroke Scale (NIHSS) score, had more often a history of coronary disease, and were less often current or recent smokers than patients with another stroke type (Table 1).

The proportion of patients with a posterior stroke location among patients with a cardiac origin of their stroke (28\%) was not significantly higher compared to those with another origin (25\%): RR 1.14; 95\% CI 0.94-1.37 (Table 3 ). Baseline characteristics with $>3 \%$ missing values (body mass index, NIHSS score and migraine) were not used for bivariable analyses as this would decrease the number of included patients substantially. None of the remaining variables had a large influence on the crude RR for posterior stroke, and therefore only age and sex were used in the multivariable analyses. As a result, the additional Poisson regression analyses to assess the influence
Table 2. Stroke etiology in all 3,311 patients with ischemic stroke or TIA

\begin{tabular}{lc}
\hline Stroke subtype & $n(\%)$ \\
\hline Atherosclerosis & $802(24)$ \\
Cardioembolism & $578(18)$ \\
Small vessel occlusion (lacune) & $623(19)$ \\
Other & $321(10)$ \\
Undetermined & $944(29)$ \\
Unknown & $43(1)$ \\
\hline
\end{tabular}

TIA, transient ischemic attack.

of these potential confounders only marginally changed the RR 1.16; 95\% CI 0.96-1.41.

When only patients with a known duration of symptoms longer than $24 \mathrm{~h}$ were included, that is, exclusion of patients with a TIA, 1,112 patients remain eligible for Poisson regression analyses. This marginally changed the proportion of patients with a posterior stroke (28\%) compared to those with another origin (30\%) in case of a cardiac source; age and sex adjusted RR 0.91; 95\% CI 0.671.24 .

With the stepwise backward regression analyses, we found that a history of coronary artery disease, higher age, and a higher NIHSS score were related to cardioembolic stroke, while current or recent smoking and hyperlipid- 
Table 3. Chance for cardioembolic etiology of ischemic stroke in case of posterior localization

\begin{tabular}{lllll}
\hline & $\begin{array}{l}\text { Cardioembolism } \\
(n=388), n(\%)\end{array}$ & $\begin{array}{l}\text { No cardioembolism } \\
(n=1,040), n(\%)\end{array}$ & $\begin{array}{l}\text { Relative risk } \\
(95 \% \mathrm{CI})\end{array}$ & $\begin{array}{l}\text { Adjusted* relative } \\
\text { risk (95\% CI) }\end{array}$ \\
\hline $\begin{array}{l}\text { Posterior stroke } \\
\text { Anterior stroke }\end{array}$ & $110(28)$ & $259(25)$ & $1.14(0.94-1.37)$ & $1.16(0.96-1.41)$ \\
\hline
\end{tabular}

* Adjusted for age and sex.

Table 4. Chance for cardioembolic etiology of ischemic stroke based on stepwise backward logistic regression

\begin{tabular}{lrl}
\hline & \multicolumn{1}{c}{ Beta } & OR (95\% CI) \\
\hline Recent or current smoking & -0.755 & $0.47(0.36-0.62)$ \\
Hyperlipidemia & -0.297 & $0.74(0.59-0.94)$ \\
NIHSS score (per point increase) & 0.058 & $1.06(1.04-1.08)$ \\
Age (per year increase) & 0.013 & $1.01(1.01-1.02)$ \\
Coronary artery disease & 0.436 & $1.55(1.17-2.05)$ \\
\hline
\end{tabular}

NIHSS, National Institutes of Health Stroke Scale.

Table 5. Chance for cardioembolic etiology of ischemic stroke based on risk score

\begin{tabular}{lll}
\hline & Risk score & Risk of cardiac source, \% \\
\hline Q1 & $<0.4633$ & 11.7 \\
Q2 & $0.4633-0.8804$ & 21.5 \\
Q3 & $0.8804-1.1917$ & 23.1 \\
Q4 & $>1.1917$ & 36.8 \\
\hline
\end{tabular}

emia were inversely related with cardioembolic stroke (Table 4). The chance for cardioembolic etiology of ischemic stroke based on the risk score ranged from $11.7 \%$ for the first quartile to $36.8 \%$ for the fourth quartile (Table 5 ). The $\mathrm{C}$-statistic (area under the curve of the receiver operating characteristic curve) was 0.65 (95\% CI 0.62-0.68) (Fig. 2).

\section{Discussion}

The goal of our study was to measure an association between a cardiac etiology of stroke and posterior stroke localization, and in addition to describe the precision of that measurement. The major finding of this study is that we could not confirm our hypothesis that a posterior localization of thromboembolic stroke is associated with a cardioembolic source for ischemic stroke. However, clas- sification of this study as strictly negative is potentially misleading, because such classification is done on basis of CIs, the magnitude of which is determined both by effect size and precision. With only 5 more cardioembolic strokes in the posterior localization, the results would have been statistically significant. However, the trend is only small, and therefore stroke localization on itself has hardly any additional value in the consideration to perform cardiac examination after ischemic stroke of undetermined source. Furthermore, the sensitivity analysis without patients with TIAs neither showed an effect. We performed this additional analysis because in these patient's radiographic conformation of infarction in the posterior circulation is not possible and in some occasions clinical symptoms of TIA are caused by nonvascular events.

A history of coronary artery disease, a higher age and NIHSS score, and lack of determinants of atherosclerosis increased the chance of a cardiac source of the stroke. Although the ability of the risk model to discriminate between patients with or without a cardiac source of ischemic stroke is only moderate, these clinical characteristics may necessitate a further exploration on a cardiac source for ischemic stroke. This is clinically relevant as in patients with a previous stroke in the presence of AF, use of antiplatelet agents will prevent 40 vascular events per 1,000 patients per year, whereas coumarins will prevent 90 vascular events per 1,000 patients per year [14]. Factor $\mathrm{Xa}$ inhibitors further boost the prevention rate with $22 \%$ (to 110) compared with coumarins [15], so estimated reduction of vascular events is 70 per 1,000 patients per year in patients detected with $\mathrm{AF}$ after ischemic stroke and in whom antiplatelet agents are changed to an oral factor Xa inhibitor. The number needed to treat, with anticoagulants, to prevent 1 vascular event per year is 9 .

Our findings are in line with a cohort study in stroke patients in which patients with carotid stenosis, other apparent stroke causes such as dissection or vasculitis, or an apparent embolic source were excluded. The authors found that multiple ischemic lesion patterns in the posterior circulation were associated with the presence of a 


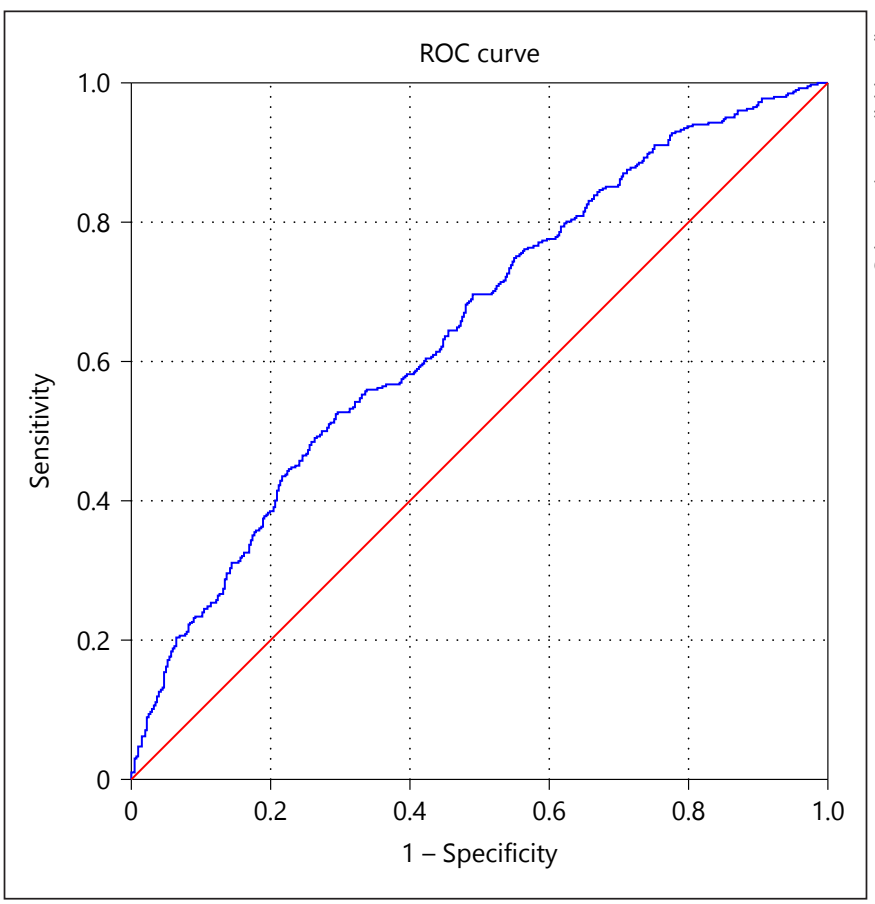

Fig. 2. ROC curve of risk score. C-statistic (AUC-ROC) is 0.65 (95\% CI 0.62-0.68). ROC, receiver operating characteristic.

PFO [16]. Based on a 99mTc-MAA Brain SPECT study, this may be caused by an increased blood flow in the posterior circulation compared with that in the anterior circulation in right-to-left shunting during a Valsalva maneuver [17]. Although PFO-related stroke may not be a good model for cardioembolism in general, to our knowledge, there are no other studies on well-established cardioembolic causes and stroke distribution exploring other plausible biological explanations.

Our findings are in line with a study that aimed to determine if lesion patterns on early diffusion-weighted imaging are associated with stroke subtypes determined by the TOAST classification [18]. In a cohort of 172 stroke patients, there were 21 in the posterior circulation ( 5 single lesions in the posterior circulation and 16 multiple lesions in both anterior and posterior circulation) of which $12(57 \%)$ were cardioembolic strokes and 6 were undetermined. They also found that single corticosubcortical lesions were associated with cardioembolic stroke. Unfortunately, our database did not describe the topography of stroke lesions in such detail, so we were unable to confirm these findings. Furthermore, they found that multiple lesions in anterior and posterior circulations and multiple lesions in multiple cerebral circulations were associated with cardioembolism. Multiple lesions in the unilateral anterior circulation and small scattered lesions in 1 vascular territory were related to large-artery atherosclerosis. A larger study in 510 patients confirmed that multiple lesions in 1 anterior circulation territory suggest large-artery atherosclerosis, while multiple lesions in multiple territories (bilateral or anterior-posterior circulation) were associated with cardiogenic embolism [19]. They did, however, not look specific to single lesions in the posterior circulation.

The findings of our study are only partly in line with our previous study in patients with thromboembolic stroke after cardiac surgery [7], as the relative risk for a posterior location for stroke after cardiac surgery compared to patients with ischemic stroke without prior cardiac surgery in that study was substantial higher: 2.09 (95\% CI 1.60-2.72) vs. 1.16 (95\% CI 0.96-1.41) in the current study. Stroke after cardiac surgery is not identical to cardioembolic stroke, but this difference may also be explained by the large number of patients in which stroke localization and etiology were undetermined in the current study, and it may well be that this was not at random, for example, exploring a cardiac source was not performed in very old patients or in those with a high NIHSS, leading to a bias by indication. Furthermore, although AF is an established risk factor for stroke in the general population, it is postulated that AF is not always the cause of cardioembolic stroke, but in part may be considered as a risk marker for stroke. This is especially the case in devicedetected subclinical AF (SCAF) which is detected in up to $40 \%$ of individuals with implantable pacemakers and defibrillators capable of long-term continuous heart rhythm monitoring $[20,21]$. Fewer than $20 \%$ individuals with SCAF who had stroke during a cohort study that enrolled 2,580 pacemaker and defibrillator patients aged $\geq 65$ years with a history of hypertension but without a history of AF had evidence of SCAF in the 30 days preceding the stroke [22]. Stroke mechanism in (particularly subclinical) AF may therefore not always be cardioembolic. However, in the Parelsnoer Institute-Cerebrovascular Accident Study Group, cardioembolism was considered definitive if patients had a history of AF or if it was detected thereafter, although based on the abovementioned findings not all strokes in AF patients necessarily had a cardiac etiology and therefore might dilute the association between cardioembolism and posterior stroke localization. The number of SCAF patients was not registered in the Parelsnoer Institute-Cerebrovascular Accident Study Group so the contribution of this diluting effect is unknown.

Our findings are based on data acquired in a large prospective nationwide cohort study enlarging the generaliz- 
ability of the results. Clinical consequences may be that in presence of posterior stroke localization in stroke with undetermined source, the threshold for performing additional cardiac examination and monitoring is slightly lowered. The need for cardiac examination may be more pressing in concomitant presence of male sex, a high NIHSS score, and no history of recent smoking and hyperlipidemia.

Phenotyping of patients with ischemic stroke of undetermined source with a higher risk for AF may increase the efficacy of additional cardiac work up. Although several parameters, including increased multiple strokes, age and male sex, are associated with AF occurrence, no clear evidence exists which (combination of) risk factors identify those patients who would benefit most from extensive cardiac monitoring. Added to known risk factors from the literature, our findings suggest that patients with posterior stroke localization in absence of determinants for atherosclerosis should be offered additional cardiac investigations. Prospective studies must confirm if in a phenotyping-based selection of patient's additional cardiac investigations for $\mathrm{AF}$ diagnosis and subsequent therapeutic intervention are indeed cost effective.

The most important limitation of our study is, in spite of the prospective nature of the cohort, the large number of patients in who stroke localization or etiology was undetermined. This underlines the challenges of performing a large multicenter prospective cohort study. However, with 1,428 patients from 8 University hospitals included in the primary analysis, the dataset is nevertheless large enough to allow sufficiently precise conclusions for an analysis that consists of a single characteristic (posterior stroke) with 13 potential confounders and 1 outcome (cardioembolic stroke) that occurred in 388 patients. The 1,955 patients included in the secondary analyses are more than enough for the backward stepwise regression analysis that consisted of 14 variables and one outcome that occurred in 578 patients and even more variables could be included in the model without overfitting it when used in future studies.

\section{Conclusion}

Localization in the posterior circulation is not clearly associated with a cardioembolic source for ischemic stroke, and therefore posterior stroke localization on itself is no strong indication for additional cardiac work up. The lack of determinants of atherosclerosis is stronger related with a cardiac source of ischemic stroke and may emphasize the need for cardiac examinations.

\section{Disclosure Statement}

The authors declare that there is no conflict of interest.

\section{Funding Sources}

There is no funding source to declare.

\section{References}

1 Freedman B, Camm J, Calkins H, Healey JS, Rosenquist M, Wang J, et al.; AF-Screen Collaborators. Screening for Atrial Fibrillation: A Report of the AF-SCREEN International Collaboration. Circulation. 2017 May; 135(19): 1851-67.

2 Gladstone DJ, Spring M, Dorian P, Panzov V, Thorpe KE, Hall J, et al.; EMBRACE Investigators and Coordinators. Atrial fibrillation in patients with cryptogenic stroke. N Engl J Med. 2014 Jun;370(26):2467-77.

3 Sanna T, Diener HC, Passman RS, Di Lazzaro V, Bernstein RA, Morillo CA, et al.; CRYSTAL AF Investigators. Cryptogenic stroke and underlying atrial fibrillation. N Engl J Med. 2014 Jun;370(26):247886.

4 Hart RG, Sharma M, Mundl H, Kasner SE, Bangdiwala SI, Berkowitz SD, et al.; NAVIGATE ESUS Investigators. Rivaroxaban for Stroke Prevention after Embolic Stroke of
Undetermined Source. N Engl J Med. 2018 Jun;378(23):2191-201.

5 Diener HC, Sacco RL, Easton JD, Granger CB, Bernstein RA, Uchiyama S, et al.; RE-SPECT ESUS Steering Committee and Investigators. Dabigatran for Prevention of Stroke after Embolic Stroke of Undetermined Source. N Engl J Med. 2019 May;380(20):1906-17.

6 Fennis TF, Compter A, van den Broek MW, Koudstaal PJ, Algra A, Koehler PJ. Is isolated aphasia a typical presentation of presumed cardioembolic transient ischemic attack or stroke? Cerebrovasc Dis. 2013;35(4):337-40.

7 Pierik R, Uyttenboogaart M, Erasmus ME, Scheeren TW, van den Bergh WM. Distribution of perioperative stroke in cardiac surgery. Eur J Neurol. 2019 Jan;26(1):184-90.

8 Nederkoorn PJ, van Dijk EJ, Koudstaal PJ, Luijckx GJ, van Oostenbrugge RJ, Visser MC, et al.; Dutch String-of-Pearls Stroke StudyGroup. The Dutch String-of-Pearls Stroke
Study: protocol of a large prospective multicenter genetic cohort study. Int J Stroke. 2015 Jan;10(1):120-2.

9 WHO MONICA Project Principal Investigators. The World Health Organization MONICA Project (monitoring trends and determinants in cardiovascular disease): a major international collaboration. J Clin Epidemiol. 1988;41(2):105-14.

10 Adams HP Jr, Bendixen BH, Kappelle LJ, Biller J, Love BB, Gordon DL, et al. Classification of subtype of acute ischemic stroke. Definitions for use in a multicenter clinical trial. TOAST. Trial of Org 10172 in Acute Stroke Treatment. Stroke. 1993 Jan;24(1): 35-41.

11 Amarenco P, Bogousslavsky J, Caplan LR, Donnan GA, Hennerici MG. New approach to stroke subtyping: the A-S-C-O (phenotypic) classification of stroke. Cerebrovasc Dis. 2009;27(5):502-8. 
12 Rovira A, Grivé E, Rovira A, Alvarez-Sabin J. Distribution territories and causative mechanisms of ischemic stroke. Eur Radiol. 2005 Mar;15(3):416-26.

13 Knol MJ, Le Cessie S, Algra A, Vandenbroucke JP, Groenwold RH. Overestimation of risk ratios by odds ratios in trials and cohort studies: alternatives to logistic regression. CMAJ. 2012 May;184(8):895-9.

14 EAFT (European Atrial Fibrillation Trial) Study Group. Secondary prevention in nonrheumatic atrial fibrillation after transient ischaemic attack or minor stroke. Lancet. 1993 Nov;342(8882):1255-62.

15 Bennaghmouch N, de Veer AJ, Bode K, Mahmoodi BK, Dewilde WJ, Lip GY, et al. Efficacy and Safety of the Use of Non-Vitamin K Antagonist Oral Anticoagulants in Patients With Nonvalvular Atrial Fibrillation and Concomitant Aspirin Therapy: A Meta-Analysis of
Randomized Trials. Circulation. 2018 Mar; 137(11):1117-29.

16 Jauss M, Wessels T, Trittmacher S, Allendörfer J, Kaps M. Embolic lesion pattern in stroke patients with patent foramen ovale compared with patients lacking an embolic source. Stroke. 2006 Aug;37(8):2159-61.

17 Hayashida K, Fukuchi K, Inubushi M, Fukushima K, Imakita S, Kimura K. Embolic distribution through patent foramen ovale demonstrated by $(99 \mathrm{~m}) \mathrm{Tc}-\mathrm{MAA}$ brain SPECT after Valsalva radionuclide venography. J Nucl Med. 2001 Jun;42(6):859-63.

18 Kang DW, Chalela JA, Ezzeddine MA, Warach S. Association of ischemic lesion patterns on early diffusion-weighted imaging with TOAST stroke subtypes. Arch Neurol. 2003 Dec;60(12):1730-4.

19 Wessels T, Wessels C, Ellsiepen A, Reuter I, Trittmacher S, Stolz E, et al. Contribution of diffusion-weighted imaging in determination of stroke etiology. AJNR Am J Neuroradiol. 2006 Jan;27(1):35-9.

20 Glotzer TV, Daoud EG, Wyse DG, Singer DE Ezekowitz MD, Hilker C, et al. The relationship between daily atrial tachyarrhythmia burden from implantable device diagnostics and stroke risk: the TRENDS study. Circ Arrhythm Electrophysiol. 2009 Oct;2(5):47480.

21 Healey JS, Connolly SJ, Gold MR, Israel CW, Van Gelder IC, Capucci A, et al.; ASSERT Investigators. Subclinical atrial fibrillation and the risk of stroke. N Engl J Med. 2012 Jan; 366(2):120-9.

22 Brambatti M, Connolly SJ, Gold MR, Morillo CA, Capucci A, Muto C, et al.; ASSERT Investigators. Temporal relationship between subclinical atrial fibrillation and embolic events. Circulation. 2014 May;129(21):2094-9. 